\title{
Growth and Investigation of L-Cysteine Doped Zinc (Tris) Thiourea Sulphate Single Crystals
}

\author{
A. David Kalaimani Raj ${ }^{1}$, S. Chidambaram ${ }^{2}$, R. Manimekalai ${ }^{3 *}$ \\ ${ }^{1,2,3}$ Department of Physics, A.V.V.M. Sri pushpam college, Poondi, Tamil Nadu, India. \\ *Corresponding Author: (R. Manimekalai) Maniabi64@gmail.com , Tel.: +919942544718
}

Available online at: www.isroset.org

Accepted: 27/Jun/2018, Online: 31/Aug/2018

\begin{abstract}
The L- Cysteine doped Zinc (tris) thiourea sulphate single crystals was grown by the slow evaporation method. The single crystal XRD analysis is used to determine the unit cell parameters of the grown L- Cysteine doped Zinc (tris) thiourea sulphate single crystals. The various functional groups of the grown crystal are determined by using FTIR spectrum. The optical properties of L- Cysteine doped ZTS crystal is determined by UV-Vis spectral studies. The Florescence spectrum of L- Cysteine doped ZTS crystal exhibits the strong emission in the visible region at $565 \mathrm{~nm}$. The SHG efficiency of the grown L-Cysteine doped Zinc (tris) thiourea sulphate single crystals was confirmed by Kurtz powder technique.
\end{abstract}

Keywords - Crystal growth, Single XRD, FTIR, Optical Studies, NLO studies, Fluorescence studies.

\section{INTRODUCTION}

The rapid growth of the modern communication industry has increased the demand for the materials with impressive physical properties. So the researchers are intent on creating new enhanced crystals. This has resulted in the growth of new non-linear optical crystals for optoelectronic applications. In material science, the crystal growth is one of the important fields which involve phase transformation. Amino acids are organic molecules that contain an amino group and a carboxyl group. The amino acids, which can be synthesized in the body, are known as non-essential amino acids. Amino acids, which cannot be synthesized in the body, but obtained by diet, are known as essential amino acids.

The amino acids are usually colorless, crystalline solids and soluble in water. L - Cysteine is one of the sulfur containing non-essential amino acids, which is found naturally in many proteins. L - Cysteine is naturally found in wool, feather and hair. Cysteine is a hydrophilic amino acid. The slow evaporation technique is a suitable method to grow crystals.

In this present work, organic (Zinc Sulphate) and inorganic (thiourea) compounds are tried, along with amino acid (L-Cysteine) to obtain a hybrid material with NLO property. In this paper, we have grown L-Cysteine doped with pure ZTS and the crystals were characterized by single
X - Ray diffraction Studies (XRD), Fourier Transform Infrared Spectroscopy (FTIR), UV-Visible, Fluorescence studies and Non-Linear Optical (NLO) studies. Hereafter, we name the L-Cysteine doped ZTS as LCZTS.

\section{RELATED WORK}

Recently, there are many papers reports the growth of novel single crystals by the chemical compounds Zinc Sulphate and Thiourea. The paper titled "Growth, Optical and Electrical Properties of zinc tris (thiourea) sulphate (ZTS) Single Crystals" by M.Loganayaki, A.Senthil and P.Murugakoothan reported the optical and electrical properties of Zinc tris thiourea single crystals. The authors R. Muraleedharan, J. Ramajothi and M. Basheer Ahamed also reported the various characterizations such as optical, nlo and mechanical properties of ZTS in the paper titled "Crystal Growth, Morphology, Spectral and Optical Studies of Tris (thiourea) Zinc Sulphate - Nonlinear Optical Material". Further, novel single crystals are grown by adding L - Lysine amino acid with ZTS was reported by J. Thomas Joseph Prakash and M. Lawrence, entitled "Growth and Characterization of Pure and L-lysine Doped Zinc (TRIS) Thiourea Sulphate Crystals". Similarly, the mechanical and optical properties of L-Serine amino acid doped pure ZTS are reported in the paper named "Investigation on the properties of L-Serine doped Zinc tris (thiourea) sulphate crystal for NLO application” by F.Helen and G. Kanchana. In this research paper, the L- Cysteine amino acid is chosen 
to develop a novel single crystal. So that, the L-Cysteine amino acid is doped with pure ZTS and the grown crystal is subjected to undergo various characterization studies such as cell parameters, optical and NLO properties. From the analyzed results, some of the characteristics of the grown crystals are given in this paper.

\section{MATERIALS AND METHODS}

\section{CRYSTAL GROWTH}

Zinc sulphate and thiourea (AR grade) were taken in the stoichiometric molar ratio 1:3 and dissolved in deionized water. The mixture was stirred well using a magnetic stirrer. The solution is placed in a dust free atmosphere in a closed beaker. The ZTS crystals were grown by the slow evaporation technique at room temperature $[2,3]$.

ZTS single crystals were synthesized by the following reaction,

\section{$\left.\mathrm{ZnSO}_{4}+3 \mathrm{CS}\left(\mathrm{NH}_{2}\right)_{2} \longrightarrow \mathrm{Zn}\left[\mathrm{CS}\left(\mathrm{NH}_{2}\right)_{2}\right)_{3}\right] \mathrm{SO}_{4}$}

After 16 - 21 days, good quality of ZTS single crystals was harvested. The grown crystals are transparent and hard. For the growth of L-cysteine doped ZTS single crystals, 0.01 mole L-cysteine was added to the solution of ZTS. The Single crystals of L-cysteine doped ZTS with good shape and size were harvested.

The photographs of the grown pure ZTS, 0.01 and 0.1 mole LCZTS crystals are shown in the figures 1 (a), 1 (b) and 1 (c) respectively.

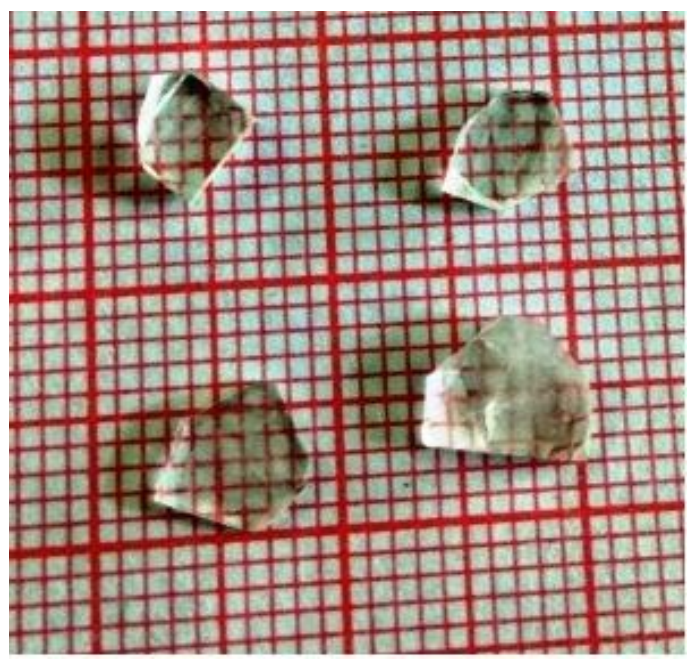

Figure -1(a) - Pure ZTS

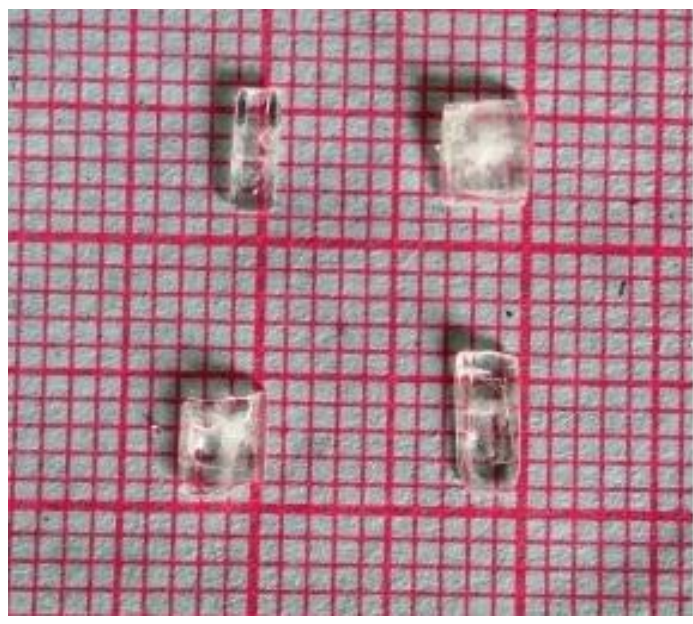

Figure - 1(b) - 0.01 M LCZTS

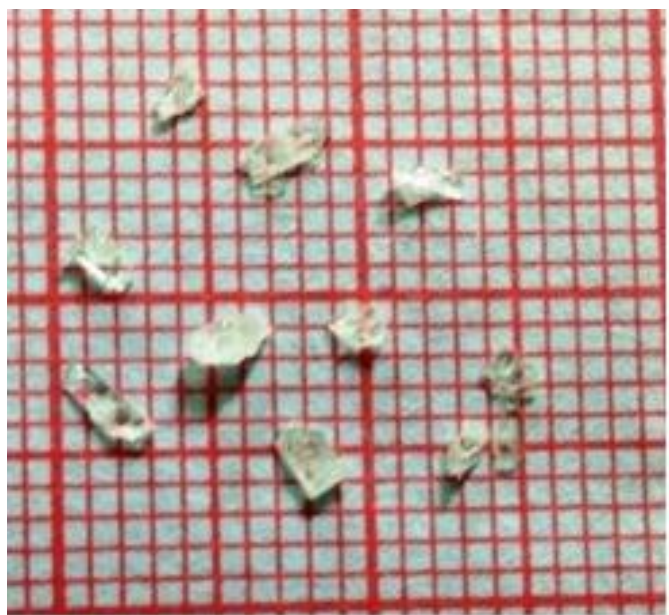

Figure - 1 (c) - 0.1 M LCZTS

In this research work, at first we tried a new semi-organic crystal in the doping ratio of $0.01 \mathrm{M}$ of L-cysteine with Zinc (tris) thiourea sulphate. But when we increase the level of doping concentration from 0.01 to $0.1 \mathrm{M}$ and so on, the size of the crystal that we get at the end is too small. They are obtained in very tiny crystals.

\section{RESUlTS AND DisCUSSION}

The grown LCZTS crystals are subjected to different characterization techniques. The grown crystals of pure ZTS and LCZTS were confirmed by single crystal Xray diffraction analysis using BRUKER SMART APEX2 diffractometer. The PERKIN ELMER RX-1 Fourier Transform Infrared spectrometer is used to identify the various functional groups in the range of $400-4000 \mathrm{~cm}^{-1}$. The optical characteristics of the crystal were analyzed in the range of 190 and $1100 \mathrm{~nm}$ using LAMBDA-35 UV-Vis spectrometer. The NLO efficiency of grown samples is 
confirmed by Nd: YAG laser. The fluorescence studies emission spectrum was recorded by VARIAN CARY ECLIPSE Fluorescence Spectrophotometer employing 150 Watts Xenon arc discharge lamp as the excitation source.

\subsection{Single crystal $X$-ray diffraction analysis}

The single crystal X-ray diffraction study is an essential one among the characteristics of the grown crystals. It is used to identify the lattice parameters, unit cell, atomic positions and the volume of the grown crystals. The LCZTS crystals were subjected to single crystal X-ray Diffraction analysis using Bruker Smart Apex2 single crystal X-ray diffractometer. A good quality crystal was subjected to the $\mathrm{X}$-ray diffraction studies. The unit cell parameters of pure and L-Cysteine doped ZTS crystals are presented in the below Table 1 [3]. From the Table 1, it is observed that, the cell parameters of LCZTS is slightly differing from pure ZTS, which may be attributed to the presence of a different mole \% of dopants in ZTS crystals. Both pure and doped crystals are found; to be orthorhombic structure. The variation in lattice parameters and volume confirms the inclusion of dopant in ZTS lattices.

Table 1: Unit cell parameters of pure ZTS and LCZTS

\begin{tabular}{|c|c|c|c|c|c|c|c|}
\hline S. & Crystal & $\mathbf{a}$ & $\mathbf{b}$ & $\mathbf{c}$ & $\boldsymbol{\alpha}=\boldsymbol{\beta}=\boldsymbol{\gamma}$ & Volume & System \\
No & & $\left(\mathbf{A}^{\circ}\right)$ & $\left(\mathbf{A}^{\circ}\right)$ & $\left(\mathbf{A}^{\circ}\right)$ & & $\left(\mathbf{A}^{3}\right)$ & \\
\hline 1. & ZTS & 7.79 & 11.14 & 15.512 & $90^{\circ}$ & 1341.6 & Orthorhombic \\
\hline 2. & LCZTS & 7.84 & 11.22 & 15.604 & $90^{\circ}$ & 1373 & Orthorhombic \\
\hline
\end{tabular}

\subsection{FTIR Spectral Studies}

The FTIR spectrum of the grown Pure ZTS and LCZTS single crystals are recorded in the range 400 - 4000 $\mathrm{cm}^{-1}$, using Perkin-Elmer spectrometer is as shown in Figure 2. The frequencies of the LCZTS are compared with that of pure ZTS. It is seen that, the recorded LCZTS spectrum have the same absorption peaks as Pure ZTS with some slight variations. The peak at 3367 and $3366 \mathrm{~cm}^{-1}$ is assigned to $\mathrm{O}-\mathrm{H}$ symmetric stretching vibration of water molecule. The symmetric stretching vibrations are observed at 3175 and $3180 \mathrm{~cm}^{-1}$ respectively [2]. The $\mathrm{C}=\mathrm{C}$ stretching vibrations for the grown crystals at $1625 \mathrm{~cm}^{-1}$. The grown crystals exhibits absorption peaks at $1400 \mathrm{~cm}^{-1}$ due to $\mathrm{C}=\mathrm{S}$ asymmetric stretching vibrations [3]. The prominent peaks near 1112 and $1106 \mathrm{~cm}^{-1}$ are attributed to N-C-N stretching. The peak at $714 \mathrm{~cm}^{-1}$ is due $\mathrm{C}=\mathrm{S}$ symmetric stretching vibrations [5]. The peak at 478 and $480 \mathrm{~cm}^{-1}$ confirms the presence of sulphate ion. S-C-N asymmetric bending is also observed at 620 and $619 \mathrm{~cm}^{-1}[3,5]$.

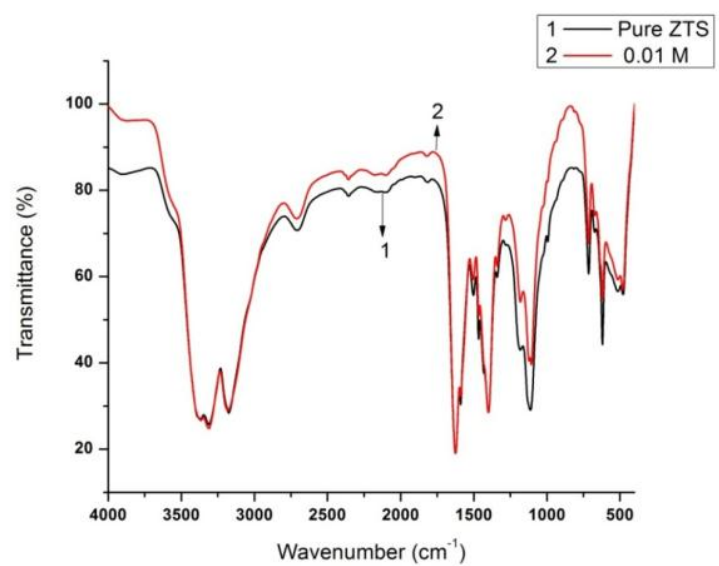

Figure - 2. FTIR Spectrums of ZTS and LCZTS

\subsection{UV - Vis Spectral Studies}

The Optical Transmission of the grown crystal are studied by using Perkin Elmer Lambda 35 UV-Visible spectrophotometer in the wavelength range of 190 to 1100 nm. The absorption spectra of the grown crystals have lower cut off wavelengths around $198.4 \mathrm{~nm}$ for Pure ZTS and $375.3 \mathrm{~nm}$ for 0.01 Mole LCZTS [2]. The absorption spectra of pure ZTS and 0.01 Mole LCZTS are shown in the following Figure 3(a). Similarly, the transmittance spectra for the pure ZTS and 0.01 M of LCZTS are shown in the Figure 3(b). From the transmittance graph, the grown crystals show good transmittance in the entire visible region. The optical band gap of the grown 0.01 Mole LCZTS was determined as $3.2 \mathrm{eV}$ using Tauc's plot $[1,5]$ as shown in Figure 3(c). The $\mathrm{ZnO}$ material has wide direct band gap (3.37 $\mathrm{eV}$ at room temperature). Therefore, it's most common potential applications are in laser diodes and light emitting diodes (LEDs). On further improvisation of the grown crystal, it may be suitable instead of $\mathrm{ZnO}$ due to the relevant energy gap in optoelectronic devices.

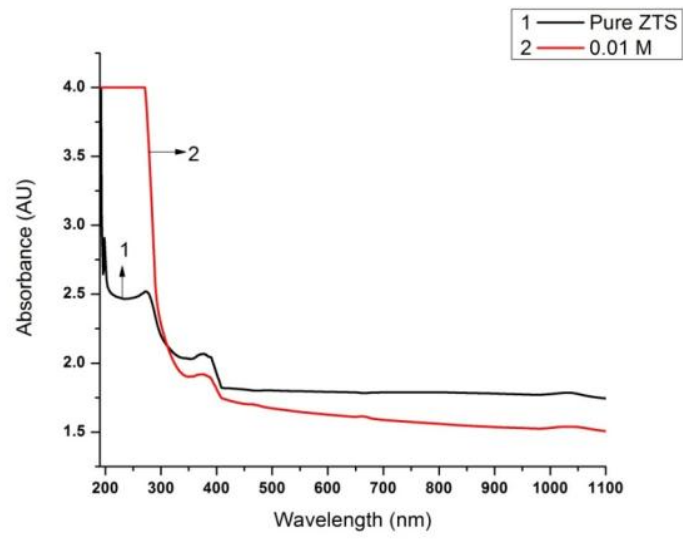

Figure - 3(a). Absorption spectrums of ZTS and LCZTS 


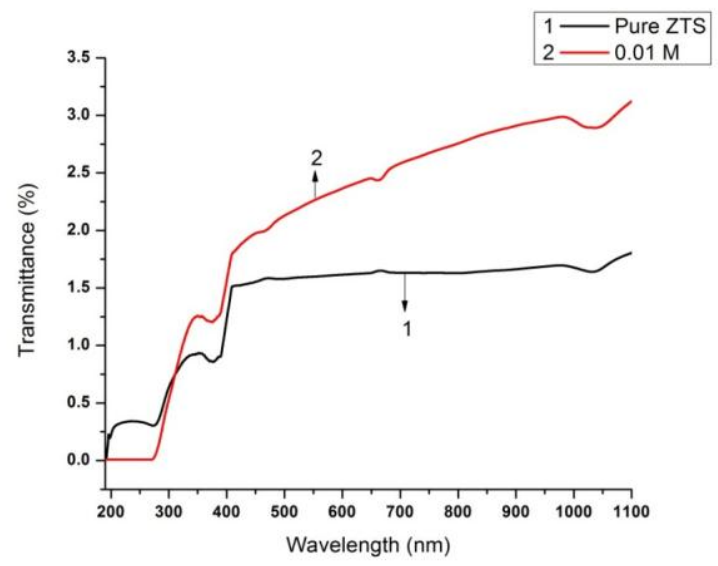

Figure - 3(b). Transmittance spectrums of ZTS and LCZTS

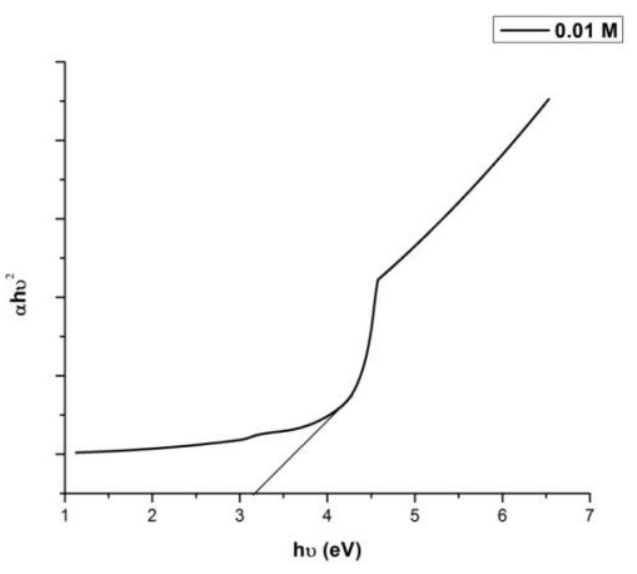

Figure - 3(c). Tauc's Plot of LCZTS

\subsection{Fluorescence Studies}

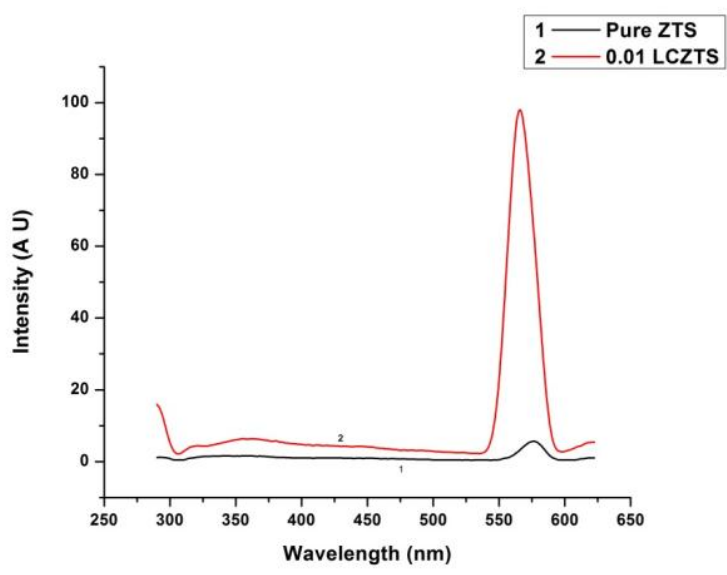

Figure - 4. Fluorescence spectrums of ZTS and LCZTS
The grown LCZTS single crystals are subjected to fluorescence studies using VARIAN CARY ECLIPSE fluorescence spectrophotometer. The Figure 4 shows the emission spectrums of pure ZTS and 0.01 mole LCZTS single crystals. The peak at $576 \mathrm{~nm}$ is observed in the emission spectrum of pure ZTS. Similarly, the peaks at $362 \mathrm{~nm}$ and $565 \mathrm{~nm}$ were observed in the emission spectrum of LCZTS [15].

\subsection{NLO Studies}

The Second harmonic generation (SHG) is a nonlinear optical process, also called as frequency doubling. Second harmonic generation (SHG) for the powder of grown LCZTS has been carried out by using Kurtz powder technique [2]. The grown crystals are crushed into a fine powder and densely packed between two transparent glass slides. A Q switched Nd: YAG laser emitting a fundamental wavelength of $1064 \mathrm{~nm}$ was allowed to strike the sample cell. The SHG output $532 \mathrm{~nm}$ (green light) was finally detected by the photomultiplier tube, in the repetition rate of $10 \mathrm{~Hz}$ with the pulse width of $6 \mathrm{~ns}$. From observed results, LCZTS crystal has received the output energy of $2.63 \mathrm{~mJ}$ from the input energy $0.70 \mathrm{~J}$. The SHG efficiency of the grown 0.01 Mole of LCZTS crystals was found to be 0.2941 times that of KDP [5].

\section{CONCLUSION AND FUture SCOPE}

The LCZTS single crystals were grown by slow evaporation technique in a period of three - four weeks. The grown crystal structure was identified as orthorhombic by the single XRD analysis. The functional groups and its vibrational modes are analyzed by the FTIR spectrum. The absorption in the entire visible range and the lower cut off wavelength for LCZTS is $375.3 \mathrm{~nm}$. The transmittance property of the grown crystal is also good. By using UV transmittance study, the optical band gap energy of the LCZTS crystal was found to be $3.2 \mathrm{eV}$. The Fluorescence spectrum of the grown LCZTS crystal shows the green emission $(565 \mathrm{~nm})$. The nonlinear optical property of the grown crystal is analyzed with Kurtz and Perry Powder technique and its efficiency was found to be 0.2941 times that of KDP. On doing further improvisation of the grown crystals, it may be suitable for optoelectronic devices.

\section{ACKNOWLEDGMENT}

The authors thank Dr. G.V. Vijayaraghavan, Associate professor, Department of Physics, B.S.Abdur Rahman Crescent Institute of Science and Technology, Chennai, India for providing SHG facilities. The authors acknowledge St. Joseph's College, Trichy, India for spectral 
facilities. The authors gratefully acknowledge the Sophisticated Analytical Instrument Facility, Indian Institute of Technology Madras, Chennai, India, for the XRD studies.

\section{REFERENCES}

[1]. R. Muraleedharan, J. Ramajothi and M. Basheer Ahamed, "Crystal Growth, Morphology, Spectral and Optical Studies of Tris (thiourea) Zinc Sulphate - Nonlinear Optical Material", Indian Journal of Science \& Technology,Vol 8(S7), pp. 165-170, 2015.

[2]. P. Malliga and C. Senthamilselvi, "Growth and Characterization of alanine doped zinc tris thiourea sulphate single crystal", RJC, Vol. 9, No. 2, pp. 144 - 148, 2015.

[3]. J. Thomas Joseph Prakash and M. Lawrence, "Growth and Characterization of Pure and L- lysine Doped Zinc (TRIS) Thiourea Sulphate Crystals", International Journal of Computer Applications, Volume 8- No.3, pp. 0975-8887, 2010.

[4]. S.Ariponnammal, S.Chandrasekaran \& C.Sanjeeviraja, "Low Temperature Photoluminescence Study on Zinc tris Thiourea sulphate Single Crystal", Digest Journal of Nanomaterials and Biostructures, Vol. 7, No. 3, pp. 947 - 957, 2012.

[5]. F.Helen \& G.Kanchana, "Investigation on the properties of $L$ Serine doped Zinc Tris Thiourea sulphate Crystal for NLO application", Indian Journal of Pure \& Applied Physics, Vol. 52, pp. 821-828, 2014.

[6]. M. Loganayaki, A. Senthil \& P. Murugakoothan, "Growth, Optical and Electrical Properties of zinc tris (thiourea) sulphate (ZTS) Single Crystals", International Journal of Computer Applications, Vol. 72, No. 1, pp. 0975 - 8887, 2013.

[7]. M. Lawrence \& J. Felicita Vimala, "Growth and characterization of pure and L-Alanine doped Zinc Tris-thiourea Sulphate (ZTS) single crystals", International Journal of Engineering. Science \& Innovative Technology,Vol.4,Iss. 2, pp. $210-218,2013$.

[8]. Hiral Baraniya \& Anand Panchal, "Growth \& Characterization of ZINC (Tris) Thiourea Sulphate (ZTS) Single Crystals”, IJET, Vol. 3, Issue. 4, pp. $2464-2467,2013$.

[9]. A. Puhal Raj and C. Ramachandra Raja, "The Effect of Phosphate Mixing on Structural, Spectroscopic, Mechanical and Optical Properties of Zinc tris Thiourea Sulphate (ZTS) Single Crystals", Journal of Minerals \& Materials Characterization \& Engineering, Vol. 11, No.5, pp. 471-478, 2012.

[10]. P. Dhanya and Fernando Loretta, "Growth and Studies on Lithium Sulphate Doped Zinc Tris Thiourea Sulphate Single Crystals", International Journal for research in Emerging Science and Technology, volume-2, issue-12, pp. 45-49, 2015.

[11]. S. Gopinath1, R. Palanivel1 \& R. Rajasekaran, "Growth and Studies of Pure and Cadmium Chloride-Doped Zinc Tris-Thiourea Sulphate (CCZTS) Crystals", International Journal of Pure and Applied Sciences and Technology, vol.1 (2), pp. 104-113, 2010.

[12]. N.Balasundari \& P.Selvarajan, "Studies on Nucleation, Growth and Characterization of L-Leucine Doped ZTS Crystals", IOSR Journal of Applied Physics, pp. 39-45, 2017.

[13]. P. Suveetha, T. Sathya, S. Sudha \& M.B. Jessie Raj, "Growth and Studies of Halides doped Zinc Tris-Thiourea Sulphate (HZTS) Crystals", International Journal of Advancements in Research \& Technology, Volume 1, Issue 5,pp. 1-7, 2012.
[14]. K.Senthil Kannan, S.Gunasekaran \& KA.Seethalakshmi, "Growth and Spectroscopic studies of Pure and L-Proline doped ZTS crystal (with Taxol)", International Journal of Scientific \& Engineering Research, Volume 4, Issue 2, pp. 1-5, 2013.

[15]. Kiran T. Rathod, I.B. Patel, C.F. Desai and Y.H. Gandhi, "PhotoLuminescence Studies of Pure and Doped ZTS Single Crystals Grown by Single Diffusion Technique”, International Journal of Luminescence and Applications, Vol. 5, No. 1, pp.86-88, 2015.

\section{AUTHORS PROFILE}

Dr. R. Manimekalai pursed M.Sc., M.Phil., and Ph.D from Bharathidasan University, Tiruchirappalli in 1988, 1989 and 2014. Presently working as an Associate professor of Physics, A.V.V.M. Sri Pushpam College, Poondi, Thanjavur-613 503, Tamil Nadu, India. Having 29 years of teaching and research experience in UG, PG and M.Phil., level. Acting as research advisor in physics from the year 2016 onwards in A.V.V.M. Sri Pushpam College (Autonomous), Poondi, Thanjavur (affiliated to Bharathidasan University). Published more than 12 research papers in reputed, peer reviewed, citation indexed international journals (Elsevier, Springer, Science direct) and international level conference. At present guiding eight Ph.D and six M.Phil research Scholars.

Mr. A. David Kalaimani Raj pursed Bachelor of Science, Master of Science and M.Phil., in A.V.V.M. Sri Pushpam College, Poondi, Thanjavur-613503, Tamil Nadu, India and Bharathidasan University, Tiruchirappalli respectively, in 2002, 2004 and 2005. $\mathrm{He}$ is currently pursuing Ph.D as a part- time research scholar in the A.V.V.M. Sri Pushpam College (Autonomous), Poondi, Thanjavur (affiliated to Bharathidasan University) and currently working as an Assistant professor in Physics, Bharathidasan University model college, Aranthangi, Pudukkottai (Dt), Tamil Nadu, India. His main research work focuses on Material science. He has 15 years of teaching and research experience in UG and PG level.

Mr. S. Chidambaram pursed Bachelor of Science and Master of Science, in Government Arts College, Udhagamandalam, Tamil Nadu, India and Bharathiar university Coimbatore respectively, in 2011, 2014, and M.Phil., in A.V.V.M. Sri Pushpam College, Poondi, Thanjavur, Tamil Nadu, India and Bharathidasan University, Tiruchirappalli respectively, in 2016. He is currently pursuing Ph.D as a Full- time research scholar in the A.V.V.M. Sri Pushpam College (Autonomous), Poondi, Thanjavur (affiliated to Bharathidasan University) Tamil Nadu, India. His main research work focuses on Material science. 